\title{
ESTRUCTURACIÓN DE UN MODELO DE NEGOCIO PARA LOS PRODUCTORES DE CACAO SOCIEDAD LA NUEVA GEMA DEL MUNICIPIO MUZO - BOYACÁ
}

\section{STRUCTURING A BUSINESS MODEL FOR COCOA PRODUCERS SOCIEDAD LA NUEVA GEMA DEL MUNICIPIO MUZO - BOYACÁ}

\section{Autor 1}

Myriam Elena Rodríguez Martínez

Economista con énfasis en Administración de empresas, Especialista en Finanzas; Maestrante en gerencia de proyectos, UNAD, Líder Semillero de investigación GEDELCO.

ORCID $000000023047670 x$

Correo electrónico: mcledesmag@unadvirtual.edu.co

\section{Autor 2}

Elías Hernández Lache

Administrador de Empresas, MG en Administración de Organizaciones, UNAD, Líder Semillero de Investigación "Lideres de Occidente".

ORCID 0000-0001-5614-5979

Correo electrónico: elias.hernandez@unad.edu.co

\section{RESUMEN}

Este artículo tiene como finalidad Estructurar un modelo de negocio para los productores de cacao de la Sociedad Agraria Cacaotera S.A.T." La nueva Gema ", del municipio de Muzo - Boyacá; para cumplir con tal fin aplica un tipo de investigación según su objetivo aplicada sus datos empleados cuantitativa y cualitativa, bajo un enfoque de investigación mixto, con el propósito de saber que tan factible a nivel de mercados, es la creación de dos líneas de productos procesados chocolate de mesa y bombón de chocolate relleno de fruta.

La propuesta de investigación se origina en la necesidad que tiene la sociedad la Nueva Gema de transformar el cacao en nuevos productos que mejoren la rentabilidad y competitividad de la sociedad. Desde una perspectiva macro, se identificó deficiencia en los ingresos para los pobladores de la zona, dentro de un proceso de transición productiva que se dio con legalización de las minas, que en consecuencia llevo a los pobladores de la zona a ver en el cacao una solución a sus problemas. El estudio de mercados permitió identificar la competencia a la que se deben enfrentar, identificar sus clientes, gusto y preferencias; lo que permitirá 
diseñar estrategias a la asociación en pro de generar ingresos rentables a la asociación cacaotera "La Nueva Gema".

Palabras clave: agroindustria; cacao; empresa; marketing; procesamiento de alimentos

\section{ABSTRACT}

The purpose of this article is to structure a business model for cocoa producers of Sociedad Agraria Cacaotera S.A.T." The new Gem ", from the municipality of Muzo - Boyacá; to fulfill this purpose, it applies a type of quantitative and qualitative research, under a mixed research approach, with the purpose of knowing how feasible at the market level the creation of two lines of is processed products: table chocolate and chocolate bonbon. Fruit filling.

The research proposal stems from the need of the New Gem society to transform cocoa into new products that improve the profitability and competitiveness of society. From a macro perspective, a deficiency in income was identified for the residents of the area, within a process of productive transition that occurred with the legalization of the mines, which consequently led the residents of the area to see cocoa as a solution to your problems. The market study allowed us to identify the competition they must face, to identify their customers, tastes and preferences; this will allow the association to design strategies in order to generate profitable income for the cocoa association "La Nueva Gema".

Keywords: Agroindustry; cacao; company; marketing; food processing.

\section{INTRODUCCIÓN}

El municipio de Muzo en el departamento de Boyacá; reconocido por las esmeraldas y fuertemente afectado por la violencia hace más de una década; encontró en el cultivo del cacao una fuente de ingresos para los pobladores de la región, por ello el objetivo del estudio es indagar mejores condiciones de vida del agricultor a través de una actividad económica rentable y sustentable; así, con el modelo de negocio se busca planificar los ingresos y beneficios para la sociedad cacaotera; buscando establecer la transformación y diversificación de los productos para atraer nuevos clientes; el presente artículo se centrará en un aparte del proyecto como es el análisis del mercado; para el proyecto de estructuración de un modelo de negocio de la sociedad agraria de cacao la nueva gema S.A.T. de Muzo. 
Lo atractivo del sector cacaotero en este caso se analiza a través de las cinco fuerzas competitivas de Porter: Ingreso de competidores, amenaza de productos sustitutos, rivalidad entre los competidores, poder de negociación con proveedores, poder de negociación con los clientes; este modelo estratégico es una herramienta que permite visualizar el desarrollo competitivo de las empresas, en este caso el análisis está centrado en el sector agroindustrial del chocolate en Colombia.

Para el caso el contenido del estudio mercado conecta con nueve elementos a saber: análisis del sector, producto, mercado potencial objetivo, investigación de mercado, oferta o competencia, demanda, canales de $\mathrm{s}$

Distribución, precio, publicidad, promoción y DOFA. El interés de la investigación radica en fortalecer y hacer viable rentable a la sociedad cacaotera La Nueva Gema del Municipio de Muzo; como eje importante de desarrollo económico y social de sus asociados y el municipio de muzo.

\section{METODOLOGÍA}

El desarrollo metodológico de la presente investigación se considera de tipo cualitativo (mediante la recolección documental, trabajo de campo e investigación participativa) y cuantitativo (mediante la aplicación de la encuesta y tabulación de los resultados obtenidos en la encuesta). Maneja las variables cuantitativas y cualitativas por tanto es Mixto, es decir, en palabras de Sampiery:

Las investigaciones con enfoque mixto consisten en la integración sistemática de los métodos cuantitativo y cualitativo en un solo estudio con el fin de obtener una "fotografía" más completa del fenómeno. Pueden ser conjuntados de tal manera que las aproximaciones cuantitativa y cualitativa conserven sus estructuras y procedimientos originales ("forma pura de los métodos mixtos"); (Chen, 2006 citado por Sampiery, 2010 p 28).

Población muestra: Para la encuesta se usó el modelo de cálculo de población infinita las personas que se les aplico la encuesta se encuentran en los rangos de edad adulta entre 20 y 60 años y a establecimientos comerciales registrados en cámara de comercio de Chiquinquirá y Tunja se estima la variación de siguiente manera

El nivel de confianza es de $0.92 \%$, con margen de error del $8 \%$, la probabilidad de éxito es del $50 \%$ y la probabilidad de no éxito es del $50 \%$ 
El error de muestreo, es decir, la desviación de la muestra seleccionada en este trabajo para aplicar la fórmula será del de $8 \%$. Este error de estimación surge a causa de observar una muestra de la población completa.

El estudio se llevó a cabo mediante de la aplicación encuestas de manera amplia, con la aplicación del programa SuerveyMonkey, compartido a una base de datos de manera electrónica y que permitió medir los hábitos de consumo y los posibles canales de distribución y el target de mercado.

\section{DISCUSIÓN}

La investigación del presente estudio busca dar solución al problema "Castellanos J (2012)" en este caso teniendo en cuenta que el estudio está dirigido a resolver la viabilidad del plan de negocios de un producto de alto impacto en mercado que resuelva necesidades de producción y comercialización de los productores, concentrando los resultados en los siguientes aspectos:

1 - Conoce el índice de consumo de productos de chocolate

2- Conocer las preferencias de consumo en los diferentes productos

3- Conocer las frecuencias de consumo

4- Conocer el nivel de aceptación de un nuevo producto

5- Conocer las líneas de productos de mayor consumo.

Se diseñaron dos encuestas una enfocado al cliente y otra a los comerciantes afiliados a las cámaras de comercio de Tunja y Chiquinquirá esta última recoge los municipios de occidente de Boyacá; esto con el fin de identificar la percepción de los dos tipos de clientes.

Inicialmente dos personas iniciaron con las encuestas, se efectuaron planes pilotos con los asociados de la " Nueva Gema" de muzo, después de probar la herramienta se aplicaron las dos encuestas; la primera se logró en un periodo de dos meses, compartiéndola mediante el programa SuerveyMonkey a través de WhatsApp y correos, donde se distribuyeron un número importante de encuesta dentro de la población objeto de investigación; en cuanto a la segunda encuesta se aplicó a los comerciantes, requiriendo de 3 meses; ya que habiendo compartido el programa por los diferentes medios ya mencionados, tan solo se lograron 20 encuestas, así que se debió contar con una persona más y realizarlas vía telefónica y así lograr la meta propuesta. 
Dentro de los principales resultados de la encuesta tenemos los siguientes: Fueron las mujeres en un $60 \%$ quienes resolvieron en gran porcentaje la encuesta; se puedo identificar que se tiene preferencia por el chocolate de mesa en un $53 \%$, la confitería a base de cacao en un $23 \%$ y el chocolate orgánico en un $16 \%$. Los resultados muestran tres indicadores relevantes sobresaliendo el chocolate de mesa, seguido de la confitería. Se puedo identificar tres ítems muy importantes para el análisis de mercados con una frecuencia de consumo diario de chocolate en un $43 \%$, entre tres y cinco veces por semana $28 \%$ y una vez al a semana $13 \%$. En cuanto a las preferencias de compra se pudo establecer que son las tiendas con un $34 \%$ las que se ubican en primer lugar seguido de supermercados con un $32 \%$ y almacenes de cadena con el $16 \%$. Este indicador da elementos importantes para definir canales de mercadeo y el direccionamiento del mercado.

Los resultados de la segunda parte de la investigación aplicada a establecimientos comerciales, en donde se indago la incidencia de ventas, preferencias de marcas de los clientes y principales proveedores; se obtuvieron resultados para el estudio de mercados identificando el $74 \%$ de los establecimientos indagados venden chocolate de mesa y el $26 \%$ no.

Entre las marcas de chocolate de mayor aceptación en los establecimientos, se encuentran tres principales indicadores: la especial con un $46 \%$, corona con un $41 \%$ y Luker con un $6 \%$ seguida de las otras marcas en un menor porcentaje, lo que permite idenficar la competencia del mercado. Los grandes proveedores son distribuidores con un $79 \%$, grupo Nutresa con un $9 \%$ y finalmente supermercados con un $7 \%$.

En cuanto a la línea confitería, el $98 \%$ de los establecimientos comerciales manifestaron vender líneas de dulces de chocolate en ella existe variedad según los clientes pero repunta la chocolatina Jet con un $18 \%$. 


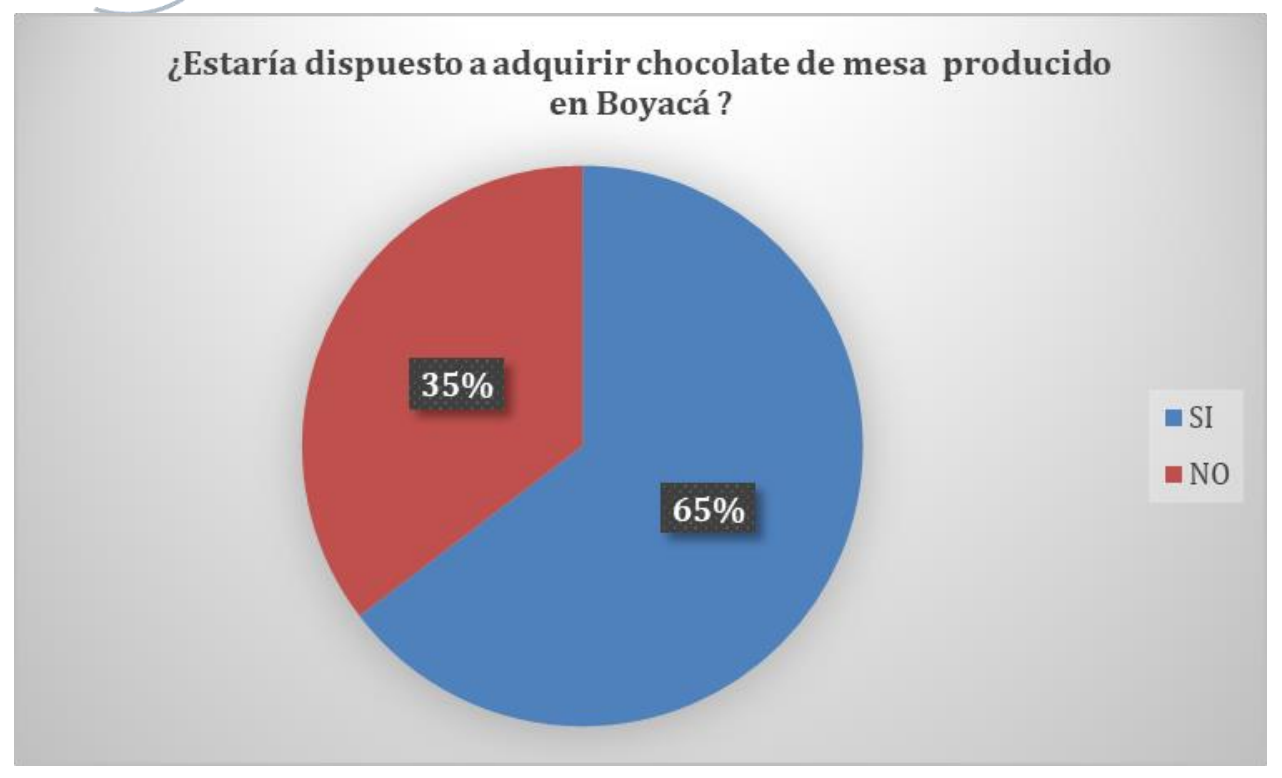

Fuente: elaboración propia

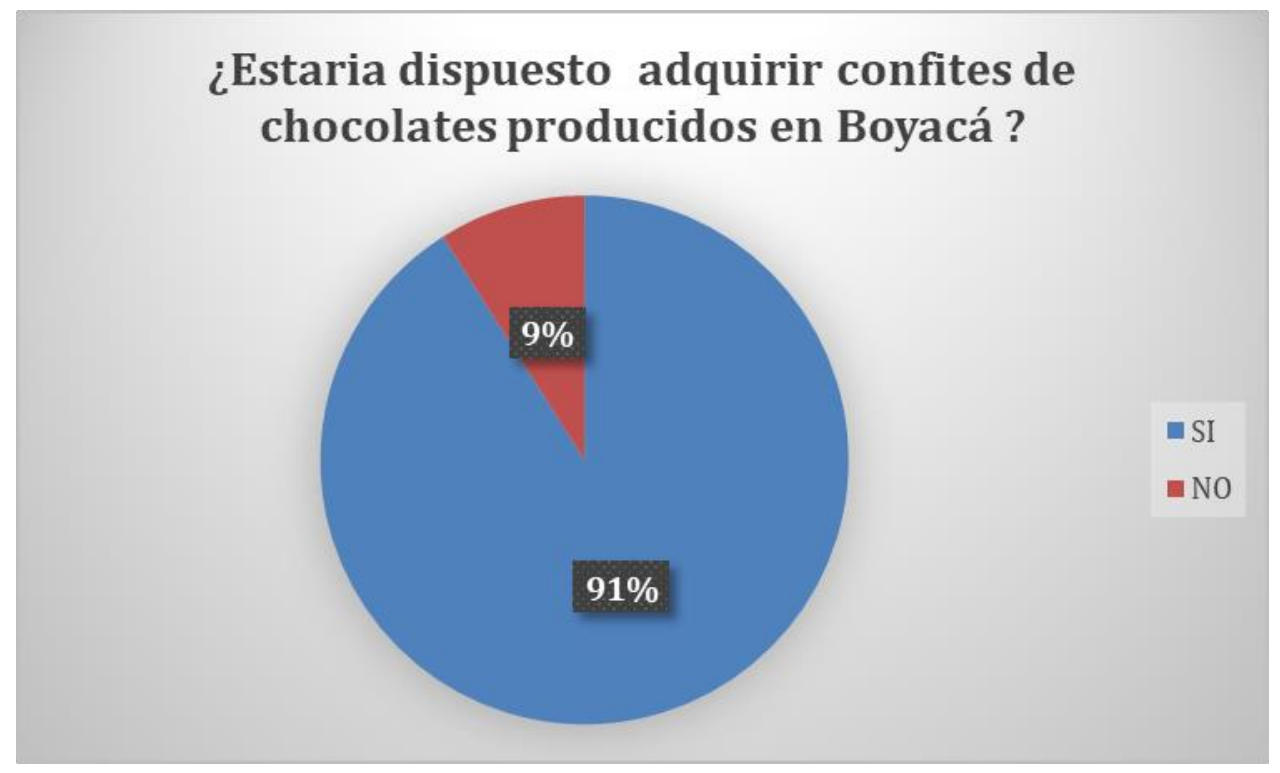

Fuente: elaboración propia

Como dato importante la población objeto de estudio manifestaron la intensión de comprar chocolate de mesa en $65 \%$ y confitería en $91 \%$ producida en Boyacá, siendo el primero de mayor consumo.Se puede inferir que estamos frente a un mercado para el producto muy atractivo, ya que la frecuencia de consumo tiene una incidnecia supremamente alta y de gran relevancia para la viabilidad del 


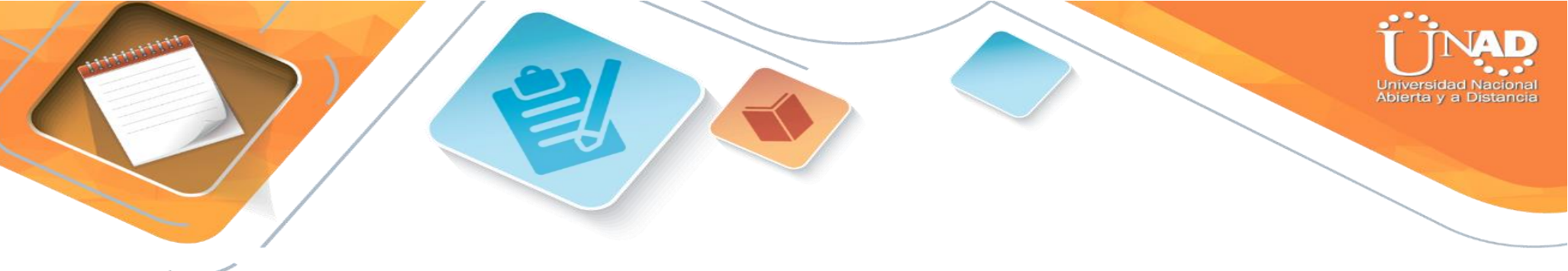

proyecto.

\section{CONCLUSIONES}

El estudio de mercados permitió identificar la competencia, el mercado objetivo, los gustos y preferencias de los clientes; que permitirán diseñar estrategias útiles para generar ingresos rentables a la asociación cacaotera "La Nueva Gema". Se puedo identificar una fuerte intención de compra a nuevos productos Boyacenses en más de un $90 \%$, muy significativo para el proyecto; siendo el chocolate de mesa y los confites los de mayor predilección.

Se puede inferir que a pesar de la fuerte competencia se cuenta con un mercado potencial con altas posibilidades de viabilidad.

\section{REFERENCIAS}

Alcaldía de Muzo Boyacá. (2019). Historia. Recuperado de http://www.muzoboyaca.gov.co/municipio/historia

Bernal, C. (2010). Metdología de la investigacion: administración, Economía, Humanidades y ciencias sociales. Bogotá, Colombia: PEARSON EDUCACIÓN,

Bernat, L., y Pinto, R. (2004). La esencia del Marketing. Cataluña, España: Universidad Politecnico de Catalunya.

Bohórquez, E. J. (2016). Plan de desarrollo más para Muso 2016-2019. Muzo, Boyacá: Alcaldía de Muzo .

Castillo, L. (2004). Análisis documental. Recuperado de https://www.uv.es/macas/T5.pdf

Córdoba, P. (2011). Formulación y evaluación de proyectos. Medellín, Colombia: Ecoe Ediciones. 
CUMP. (2019). ¿ES lo mismo Publicidad y promoción? Recuperado de https://www.cump.edu.mx/art\%C3\%ADculos-de-inter\%C3\%A9s/item/504-es-lomismo-publicidad-y-promoci\%C3\%B3n

Murcia J y Vargas E (2018). Plan de negocios para la puesta en marcha de la planta piloto para el procesamiento y transformación de cacaos finos en Santander. Tesis. Recuperado http://noesis.uis.edu.co/bitstream/123456789/12377/1/134049.pdf

Sampiery, R.H., Fernández y C., Baptista, P. (2010). Metodología de la investigación. México: Mac Graw Hill.

Porter, M. (2008). Las cinco fuerzas de competitivas que le dan forma a la estrategia. Harvard Business Revieu. América Latina. Recuperado de https://utecno.files.wordpress.com/2014/05/las_5_fuerzas_competitivas_michael_porter-libre.pdf 